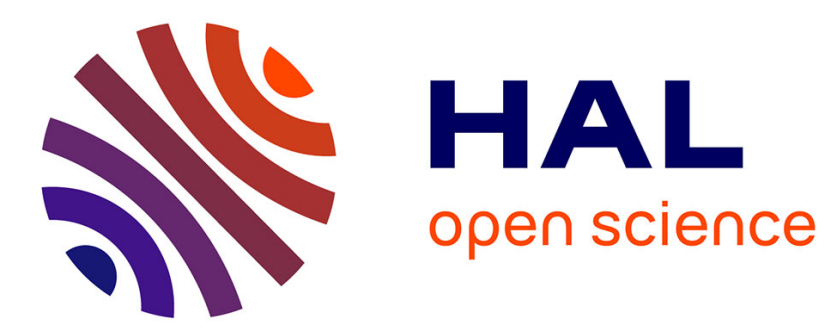

\title{
Spectres d'absorption dans l'infrarouge lointain du toluène des ortho-méta et paraxylènes a la température de l'azote liquide
}

B. Wyncke, J. Pierron, X. Gerbaux, A. Hadni

\section{- To cite this version:}

B. Wyncke, J. Pierron, X. Gerbaux, A. Hadni. Spectres d'absorption dans l'infrarouge lointain du toluène des ortho-méta et paraxylènes a la température de l'azote liquide. Revue de Physique Appliquée, 1974, 9 (2), pp.479-482. 10.1051/rphysap:0197400902047900 . jpa-00243806

\section{HAL Id: jpa-00243806 https://hal.science/jpa-00243806}

Submitted on 1 Jan 1974

HAL is a multi-disciplinary open access archive for the deposit and dissemination of scientific research documents, whether they are published or not. The documents may come from teaching and research institutions in France or abroad, or from public or private research centers.
L'archive ouverte pluridisciplinaire HAL, est destinée au dépôt et à la diffusion de documents scientifiques de niveau recherche, publiés ou non, émanant des établissements d'enseignement et de recherche français ou étrangers, des laboratoires publics ou privés. 


\title{
SPECTRES D'ABSORPTION DANS L'INFRAROUGE LOINTAIN DU TOLUÈNE DES ORTHO-MÉTA ET PARAXYLĖNES A LA TEMPÉRATURE DE L'AZOTE LIQUIDE
}

\author{
B. WYNCKE, J. PIERRON, X. GERBAUX et A. HADNI \\ Université de Nancy I, 54000 Nancy, France
}

(Reçu le 16 juillet 1973, révisé le 19 octobre 1973)

\begin{abstract}
Résumé. - A partir des diagrammes de Debye-Scherrer publiés par Biswas et Sirkar, on peut connaître le système cristallin et les dimensions de la maille primitive d'où le nombre de molécules et le groupe d'espace. On en déduit le nombre de vibrations externes actives en absorption infrarouge et en diffusion Raman. Il est intéressant de disposer de ces spectres pour confirmer ou infirmer la structure de la maille suggérée par les diagrammes. Pour le toluène, la maille serait orthorhombique $\left(\mathrm{C}_{2 \mathrm{v}}^{7}\right)$ avec 4 molécules en position générale et l'on attendrait 15 vibrations externes actives en infrarouge. Le spectre infrarouge présente 4 bandes larges qui seraient l'enveloppe des 15 raies attendues. Pour le métaxylène, la maille serait aussi orthorhombique $\left(D_{2}^{3}\right)$ avec 4 molécules en position générale, d'où encore 15 vibrations externes actives en infrarouge. Le spectre infrarouge présente 3 bandes larges qui en seraient de même l'enveloppe. Pour l'orthoxylène la maille serait orthorhombique $\left(\mathrm{D}_{2 \mathrm{~h}}^{1}\right)$ avec 4 molécules ayant un plan de symétrie localisé dans un plan de la maille, d'où 6 vibrations externes actives en infrarouge. On en observe cinq ce qui confirme la structure et permet de rejeter une autre possibilité avec 8 molécules dans la maille, qui conduit à 15 vibrations actives en infrarouge.

Enfin, pour le paraxylène, la maille serait monoclinique $\left(C_{2 h}^{2}\right)$, la molécule aurait un centre de symétrie, et ce centre serait situé sur un centre de symétrie de la maille et il y aurait 2 molécules par maille primitive. D'où 3 vibrations externes de translation actives en infrarouge et 6 vibrations actives en Raman. L'expérience donne effectivement 3 raies d'absorption à 38,56 et $76 \mathrm{~cm}^{-1}$ qu'on ne trouve pas dans le spectre Raman $\left(70,93,114\right.$ et $\left.142 \mathrm{~cm}^{-1}\right)$ publié par Kondilenko, Korotkov et Litvinov. Au contraire si l'on suit Biswas et suppose les molécules en position générale, il faut admettre qu'il y a 4 molécules dans la maille et qu'il n'y a pas de règle d'exclusion, ce qui est contraire aux observations.
\end{abstract}

Abstract. - From the Debye-Scherrer diagrams published by Biswas and Sirkar, the lattice system and the primitive cell dimension are known and hence the number of molecules and the space group. Then the number and symmetry of infrared- and Raman-active vibrations are deduced. These spectra should be measured to confirm the crystal structure which had been suggested by the $\mathrm{X}$ Ray data. For toluene, the Bravais cell should be orthorhombic $\left(\mathrm{C}_{2 \mathrm{v}}^{7}\right)$ with 4 molecules in general position, and 15 infrared-active external vibrations should be expected. The far infrared spectrum shows 4 wide bands at 29, 42, 72, and $106 \mathrm{~cm}^{-1}$, which should be their envelope. For metaxylene the primitive cell should also be orthorhombic $\left(D_{2}^{3}\right)$ with 4 molecules in general position, hence again 15 infrared-active external vibrations. The far infrared spectrum shows three wide bands at 49,56, $98 \mathrm{~cm}^{-1}$ which should be their envelope. For orthoxylene the situation is similar with again an orthorhombic 4-molecule unit cell $\left(\mathrm{D}_{2 \mathrm{~h}}^{1}\right)$, but the molecules have a plane of symmetry located parallel to one of the unit cell. Hence there should be only 6 infrared-active external vibrations. Five of them are clearly observed, which is a good check since the other possibility with 8 molecules in the unit cell should lead to 15 infrared-active external vibrations and a broad envelope.

Finally, the case of paraxylene should be similar to the case of naphthalene. The unit cell is monoclinic $\left(\mathrm{C}_{2 \mathrm{~h}}^{2}\right)$, the molecule has a center of symmetry and this center is located on a center of symmetry of the primitive cell. Hence it contains only two molecules, implying 3 infrared-active translational vibrations and 6 Raman-active rotational vibrations. Indeed, three absorption lines are observed at 38,56 , and $76 \mathrm{~cm}^{-1}$, well separated from the Raman lines which have been described by Kondilenko et al., at 70, 93,114, and $142 \mathrm{~cm}^{-1}$. On the other hand if we should accept the assumption of Biswas that the molecules are in general positions, there should be 4 molecules in the Bravais cell with no exclusion rule between infrared and Raman lines, which is contrary to our observations. 
Introduction. - L'étude d'un monocristal de benzène en lumière polarisée avait permis de situer entre 56 et $102 \mathrm{~cm}^{-1}$ les 6 raies de translation qu'on pouvait attendre de la symétrie de la maille orthorhombique de 4 molécules [1]. Il était intéressant de prolonger cette étude sur le toluène (1 groupement méthyle) et sur les trois xylènes (2 groupements méthyle). L'abaissement de la symétrie permet de prévoir plus de vibrations actives en absorption. Toutefois nous n'avons pas pu opérer sur des monocristaux, èt nous avons utilisé de la lumière infrarouge naturelle. Les spectres ne sont que partiellement résolus mais ils apportent tout de même des renseignements intéressants.

1. Expérimentation. - Les produits que nous avons utilisés sont des produits " purs » achetés chez Prolabo. Le tableau I rappelle les températures normales d'ébullition et de fusion, la structure, et résume la symétrie des vibrations dont nous allons discuter. Les spectres d'absorption infrarouge sont donnés par un spectromètre à réseau Cameca SI 36 équipé d'un cryostat à température variable. Les produits sont introduits à l'état liquide dans la cuve et le refroidissement à $80 \mathrm{~K}$ permet d'obtenir des parties monocristallines dont les surfaces varient de 0,1 à $0,5 \mathrm{~cm}^{2}$ dans une cuve de diamètre $25 \mathrm{~mm}$. Nous n'utilisons que la partie centrale $(\varnothing 16 \mathrm{~mm})$ afin de ne pas être gênés par la diminution de volume qui accompagne la solidification et produit un large ménisque à la partie supérieure de la cuve.
Le tableau II donne l'ensemble des fréquences observées pour le toluène et les trois xylènes. On a rappelé les fréquences du benzène dont le spectre avait été complètement résolu [1].

\section{TABLEAU II}

Bandes d'absorption observées dans les spectres infrarouge lointain du benzène, du toluène et des xylènes à la température de $80 \mathrm{~K}$.

\begin{tabular}{|c|c|c|c|c|}
\hline \multirow[t]{2}{*}{ Benzène } & Toluène & o-xylène & m-xylène & p-xylène \\
\hline & $29 \mathrm{~cm}^{-1}$ & & & \\
\hline $56\left(b_{3 u}\right) T_{b}^{\prime}$ & & 56 & 56 & $56\left(\mathrm{~T}^{\prime}\right)$ \\
\hline $67\left(b_{2 u}\right) T_{c}^{\prime}$ & & 62 & & \\
\hline $75\left(b_{1 u}\right) T_{b}^{\prime}$ & 72 & 75 & & $76\left(\mathrm{~T}^{\prime \prime}\right)$ \\
\hline $87\left(b_{1 u}\right) T_{c}^{\prime}$ & & & & \\
\hline $99\left(\mathrm{~b}_{2 \mathrm{u}}\right) \mathrm{T}_{\mathrm{a}}^{\prime}$ & & 92 & 98 & \\
\hline \multirow[t]{3}{*}{$102\left(b_{3 u}\right) T_{a}^{\prime}$} & 106 & 101 & & \\
\hline & & 172 & & \\
\hline & 232 & 192 & 211 & 193 \\
\hline
\end{tabular}

2. Toluène. - La transmission du toluène dans l'infrarouge lointain est représentée sur la figure 1. Les maximums d'absorption se situent à $29,42,72$, 106 et $232 \mathrm{~cm}^{-1}$ à la température de l'azote liquide. A l'état liquide $(300 \mathrm{~K})$ nous ne retrouvons qu'une raie à $213 \mathrm{~cm}^{-1}$, qui correspond à celle à $232 \mathrm{~cm}^{-1}$ du solide à $80 \mathrm{~K}$. On a donc démontré son origine interne. Par contre les maximums à 29, 42, 72 et

TABLEAU I

Propriétés physiques et vibrations externes actives en infrarouge

\begin{tabular}{|c|c|c|c|c|c|c|c|c|c|c|c|c|c|}
\hline & \multirow[b]{2}{*}{$\mathrm{pM}$} & \multirow[b]{2}{*}{$\mathrm{pf}$} & \multirow[b]{2}{*}{ pe } & \multicolumn{6}{|c|}{ Structure } & \multicolumn{2}{|c|}{ Activité IR } & \multicolumn{2}{|c|}{ Densité } \\
\hline & & & & & $\mathbf{a}$ & $\mathrm{b}$ & c & $\beta$ & $\mathbf{Z}$ & $\mathrm{T}$ & $\mathbf{R}$ & liquide & solide \\
\hline $\begin{array}{l}\text { Benzène } \\
\mathrm{C}_{6} \mathrm{H}_{6}\end{array}$ & $\overline{78,11}$ & $\overline{5^{\circ}} 5$ & $\overline{80^{\circ}} 1$ & $\begin{array}{c}\text { orth. } \\
P_{\text {bca }}\left(\mathrm{D}_{2 h}\right) \\
\text { réf. [8] }\end{array}$ & $\overline{7,46}$ & $\overline{9,66}$ & $\overline{7,03}$ & - & $\overline{4}$ & $\begin{array}{r}2 b_{1 u}+2 b_{2 u}+ \\
+2 b_{3 u}\end{array}$ & - & $0, \overline{879}$ & $\overline{1,059}$ \\
\hline $\begin{array}{l}\text { Toluène } \\
\mathrm{C}_{7} \mathrm{H}_{8}\end{array}$ & 92,13 & $-95^{\circ}$ & $110^{\circ} 6$ & $\begin{array}{c}\text { orth. } \\
\mathrm{P}_{\mathrm{mn} 2_{1}}\left(\mathrm{C}_{2 \mathrm{v}}^{7}\right) \\
\text { réf. }[1]\end{array}$ & 7,33 & 8,60 & 9,20 & & 4 & $\begin{array}{r}2 a_{1}+2 b_{1}+ \\
+2 b_{2}\end{array}$ & $\begin{array}{r}3 a_{1}+3 b_{1}+ \\
+3 b_{2}\end{array}$ & 0,867 & 1,053 \\
\hline $\begin{array}{l}\text { o-xylène } \\
\mathrm{C}_{8} \mathrm{H}_{10}\end{array}$ & 106,16 & $-25^{\circ}$ & $144^{\circ}$ & $\begin{array}{l}\text { orth. } \\
\mathrm{P}_{\mathrm{mmm}}\left(\mathrm{D}_{2 \mathrm{~h}}^{1}\right) \\
\text { réf. [4] }\end{array}$ & 8,77 & 10,20 & 14,55 & & 8 & $\mid \begin{array}{r}2 b_{1 u}+2 b_{2 u}+ \\
+2 b_{3 u}\end{array}$ & $\left\{\begin{array}{r}3 b_{1 u}+3 b_{2 u}+ \\
+3 b_{3 u}\end{array}\right.$ & 0,897 & 1,081 \\
\hline $\begin{array}{l}\text { m-xylène } \\
\mathrm{C}_{8} \mathrm{H}_{10}\end{array}$ & 106,16 & $-47^{0} \cdot 4$ & $139^{\circ}$ & $\begin{array}{c}\text { orth. } \\
P_{22_{1} 2}\left(D_{2}^{3}\right) \\
\text { réf. [4] }\end{array}$ & 7,77 & 8,45 & 10,47 & & 4 & $\mid \begin{array}{r}2 b_{1}+2 b_{2}+ \\
+2 b_{3}\end{array}$ & $\mid \begin{array}{r}3 b_{1}+3 b_{2}+ \\
+3 b_{3}\end{array}$ & 0,868 & 1,023 \\
\hline $\begin{array}{l}\mathrm{p} \text {-xylène } \\
\mathrm{C}_{8} \mathrm{H}_{10}\end{array}$ & 106,16 & $13^{\circ}-14^{\circ}$ & $138^{\circ}$ & $\begin{array}{c}\text { mono. } \\
\mathrm{P}_{21 / \mathrm{m}}\left(\mathrm{C}_{2 \mathrm{~h}}^{2}\right) \\
\text { réf. [4] }\end{array}$ & 7,56 & 8,45 & 11,11 & $98^{\circ} 57^{\prime}$ & 4 & $2 a_{u}+1 b_{u} \mid$ & $3 a_{u}+3 b_{u}$ & 0,860 & 1,003 \\
\hline
\end{tabular}

$\mathrm{pM}=$ poids moléculaire pf $=$ point de fusion

pe $=$ point d'ébullition orth. $=$ orthorhombique

mono. = monoclinique

IR = Infrarouge

$\mathrm{T}=$ Translation

$\mathrm{R}=$ Rotation. 


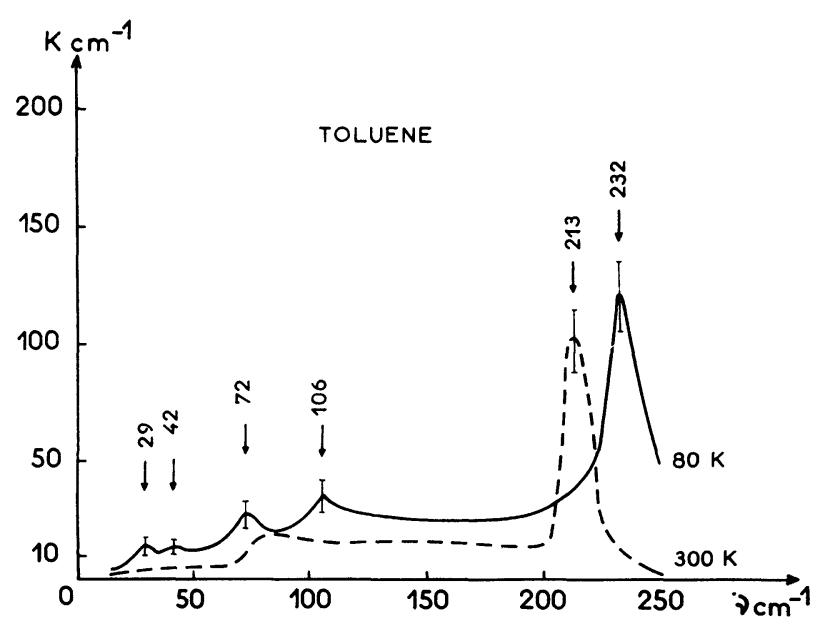

FIG. 1. - Coefficient d'absorption du toluène à l'état liquide $(300 \mathrm{~K})$ et à l'état solide polycristallin $(80 \mathrm{~K})$.

$106 \mathrm{~cm}^{-1}$ peuvent être attribués à des modes de réseau.

A $80 \mathrm{~K}$ le toluène cristallise dans le système orthorhombique avec le groupe d'espace $P_{m n 2_{1}}\left(C_{2 v}^{7}\right)$ et possède 4 molécules par maille [2]. Bien que l'on ne connaisse pas la structure on peut penser que la molécule de toluène ne possède pas d'éléments de symétrie par suite de la présence du groupement méthyle- $\mathrm{CH}_{3}$ et en particulier, si toutefois la molécule possédait un plan de symétrie normal au plan du noyau, cet élément ne serait pas commun avec un des éléments de symétrie de la maille. La molécule étant en position générale les 21 vibrations externes se répartissent en $5 a_{1}+6 a_{2}+5 b_{1}+5 b_{2}$, et seules les 15 vibrations $a_{1}, b_{1}$ et $b_{2}$ sont actives en infrarouge (et d'ailleurs en Raman). Toutefois il n'y a pas ici de séparation entre les vibrations de translation et de rotation.

En effet les 9 vibrations de translation se répartissent en $2 a_{1}+3 a_{2}+2 b_{1}+2 b_{2}$ et les 12 librations en $3 a_{1}+3 a_{2}+3 b_{1}+3 b_{2}$ en sorte que l'on ne peut dire à quels types de mouvements les absorptions à 29, 42, 72 et $106 \mathrm{~cm}^{-1}$ correspondent. Par ailleurs, nous n'observons que 4 bandes d'absorption alors que l'on en attendait 15 , le spectre n'est donc que très partiellement résolu. Il faudrait étudier un monocristal en lumière polarisée pour trouver les 15 bandes. Il est toutefois difficile de transporter un monocristal dans la cuve du spectromètre. Par analogie avec le spectre du benzène monocristallin, qui contient aussi 4 molécules par maille, on serait tenté d'attribuer un caractère translationel plus marqué aux raies du toluène à 72 et $106 \mathrm{~cm}^{-1}$.

3. Xylènes. - Les spectres de transmission des ortho-méta et paraxylènes sont représentés sur les figures 2,3 et 4 . Comme pour le toluène, les bandes de fréquences inférieures à $150 \mathrm{~cm}^{-1}$ se retrouvent à l'état liquide et sont dues à des vibrations internes.

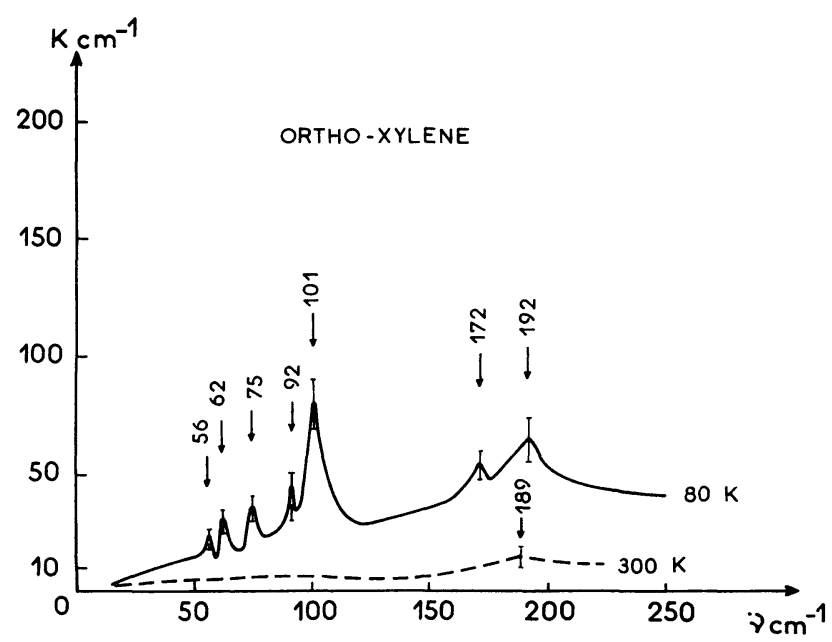

Fig. 2. - Coefficient d'absorption du paraxylène à l'état liquide $(300 \mathrm{~K})$ et à l'état solide polycristallin $(80 \mathrm{~K})$.

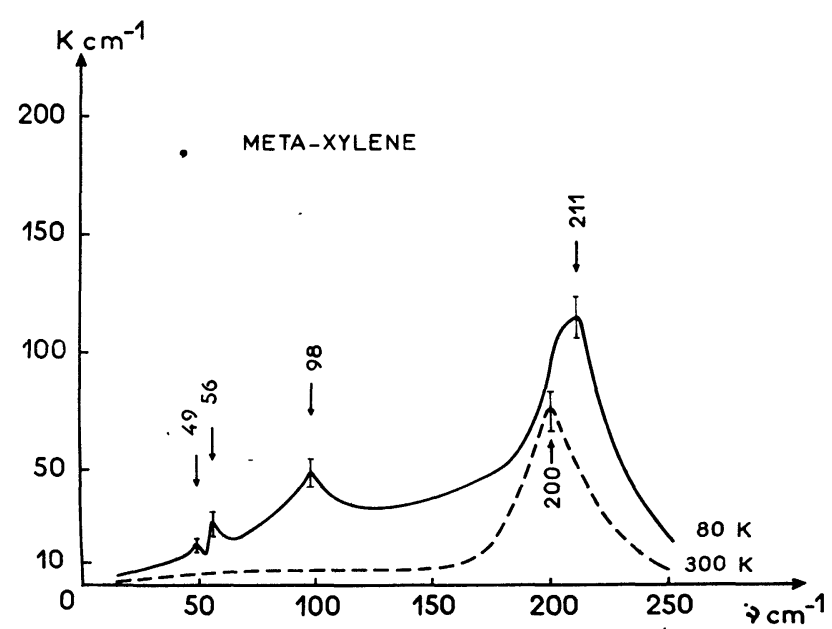

Fig. 3. - Coefficient d'absorption de l'orthoxylène à l'état liquide $(300 \mathrm{~K})$ et à l'état solide polycristallin $(80 \mathrm{~K})$.

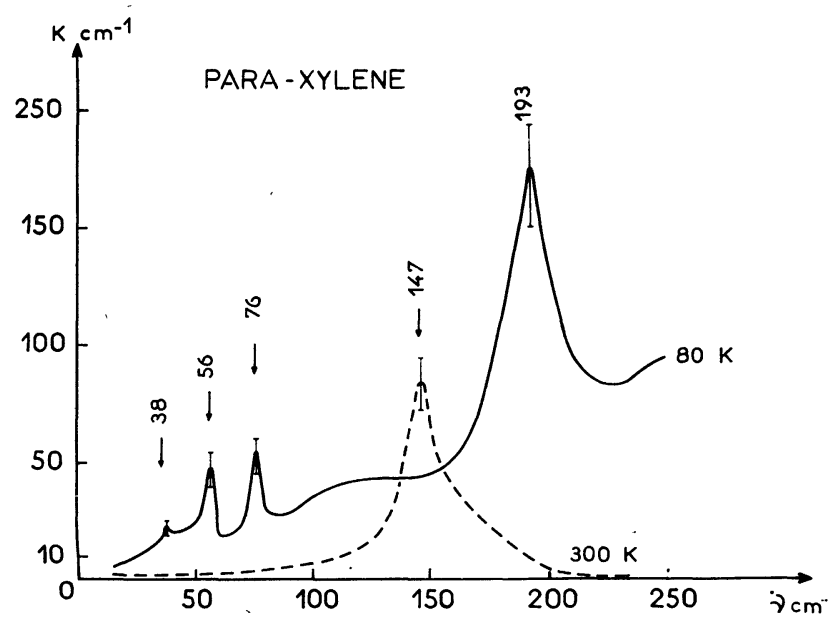

Fig. 4. - Coefficient d'absorption du métaxylène à l'état liquide $(300 \mathrm{~K}$ et à l'état solide polycristallin $(80 \mathrm{~K})$. 
3.1 ORTHOXYLÈNE. - L'orthoxylène cristallise dans le système orthorhombique avec le groupe d'espace $\mathrm{D}_{2 \mathrm{~h}}^{1}$. Il y aurait 8 molécules par maille [3].

La molécule est en position générale et aucun des éléments de symétrie que la molécule pourrait posséder ne coïncide avec les éléments de symétrie de la maille. Les vibrations externes actives en absorption infrarouge se répartissent en $5 b_{1 u}+5 b_{2 u}+5 b_{3 u}$ parmi lesquelles on distingue $2 b_{1 u}+2 b_{2 u}+2 b_{3 u}$ translations et $3 b_{1 u}+3 b_{2 u}+3 b_{3 u}$ rotations. On ne peut comme dans le cas du toluène séparer translations et rotations. Par ailleurs en Raman on attend $6 a_{g}+6 b_{1 g}+6 b_{2 g}+6 b_{3 g}$. En absorption infrarouge nous observons des maximums d'absorption à $56,62,75,92$ et $101 \mathrm{~cm}^{-1}$ qui peuvent être attribués à des vibrations externes. Ces raies sont fines et ne peuvent être expliquées comme enveloppe de plusieurs modes à l'exception peut-être de celle à $101 \mathrm{~cm}^{-1}$. On est donc conduit à penser que la cellule élémentaire ne contiendrait que 4 molécules ce qui permettrait l'activité de 6 vibrations en infrarouge au lieu de 15. Kondilenko et al. [4] aboutissent à la même conclusion car ils n'observent que 6 fréquences à 85 , $91,100,106,109$ et $140 \mathrm{~cm}^{-1}$ alors qu'ils en attendaient 24 .

3.2 MÉTAXYLÈnE. - Le métaxylène appartient également au système orthorhombique avec 4 molécules par maille [3], et possède le groupe d'espace $\mathrm{P}_{2_{12} 2_{12}}\left(\mathrm{D}_{2}^{3}\right)$. Tout comme l'orthoxylène on ne peut séparer translations et rotations car l'éventuel axe de symétrie de la molécule ne coïncide avec aucun élément de symétrie de la maille. Le spectre d'absorption infrarouge (Fig. 3 ) ne révèle que 3 bandes d'absorption à 49,56 et $98 \mathrm{~cm}^{-1}$ (on attend $5 \mathrm{~b}_{1}+5 \mathrm{~b}_{2}+5 \mathrm{~b}_{3}$ ). Ces raies sont larges. L'étude d'un monocristal en lumière polarisée montrerait très certainement que le maximum d'absorption à $98 \mathrm{~cm}^{-1}$ possède plusieurs composantes de polarisation différentes, en Raman on attend $5 b_{1}+5 b_{2}+5 b_{3}+6 a_{1}$. Kondilenko et al. [4] ont observé des raies de diffusion à 62,88 , 93, 100, 107 et $117 \mathrm{~cm}^{-1}$.

3.3 PARAXYLÈNe. - D'après Biswas [3] le paraxylène à $80 \mathrm{~K}$ présenterait une structure monoclinique avec 4 molécules en position générale. Toutefois le spectre d'absorption (Fig. 4) donne 3 raies de réseau à 38,56 et $76 \mathrm{~cm}^{-1}$ qui sont différentes des raies observées dans la diffusion de Raman à 64 , 69, 88, 94 et $108 \mathrm{~cm}^{-1}$ chez Julien-Laferrière et Lebas [5] et à 70, 93, 114 et $142 \mathrm{~cm}^{-1}$ chez Kondilenko [4]. Nous suggerons que la structure est analogue à celle du naphtalène [6] ou du paradichlorobenzène [7], c'est-à-dire monoclinique avec 2 molécules seulement dans la maille primitive. On attend alors 6 raies de libration actives en diffusion Raman et 3 raies de translation actives en absorption, et que nous situons donc à 38,56 et $76 \mathrm{~cm}^{-1}$. Une telle structure avait déjà été suggérée [5] à partir des spectres infrarouge et Raman des vibrations internes qui sont entièrement différents. Le spectre Raman des basses fréquences, connu depuis peu [4] donne des raies de plus grande fréquence : 70, 93, 114 et $142 \mathrm{~cm}^{-1}$ et confirme cette hypothèse.

4. Conclusion. - La présence des groupements méthyles additionnés au noyau benzénique dans le toluène et les xylènes abaissent considérablement la symétrie de la molécule et de la maille cristalline à l'exception du paraxylène. Il n'est plus possible alors de distinguer translations et rotations comme dans le cas du benzène où le centre de symétrie de la molécule permet d'observer en absorption infrarouge uniquement les translations et en diffusion de Raman les librations.

Les paramètres des mailles cristallines sont assez voisins et les fréquences de translation observées dans le benzène solide se retrouvent partiellement dans le toluène et les xylènes. Au point de vue analytique, la fréquence $56 \mathrm{~cm}^{-1}$ semble caractéristique des xylènes et ne se retrouve pas dans le toluène. Le domaine $90-100 . \mathrm{cm}^{-1}$ doit comporter à la fois des mouvements de translation et de rotation. Sans une connaissance plus approfondie de la structure nous ne pouvons pas attribuer définitivement ces fréquences. Par ailleurs, on attribue définitivement à une vibration interne les fréquences suivantes observées à $300 \mathrm{~K}$ pour les liquides : $213 \mathrm{~cm}^{-1}$ pour le toluène ; $200 \mathrm{~cm}^{-1}$ pour le métaxylène $; 147 \mathrm{~cm}^{-1}$ pour le paraxylène. Leur fréquence augmente de plus de $10 \%$ lors de la solidification qui rapproche considérablement les molécules (Tableau I, dernière colonne). Le cas du paraxylène est assez extraordinaire $(25 \%)$ pour être signalé. La bande en question serait due à une vibration angulaire des groupements méthyles [5].

\section{Bibliographie}

[1] Wyncke, B. et Hadni, A., C. R. Hebd. Séan. Acad. Sci. Paris 275B (1972) 825.

[2] Biswas, S. G. and Sirkar, S. C., Ind. J. Phys. 31 (1957) 141.

[3] Biswas, S. G., Ind. J. Phys. 34 (1960) 263.

[4] Kondilenko, I. I., Korotkov, P. A. and Litvinov, G. S., Optics and Spectroscopy 32 (1972) 484.
[5] Julien-Laferrière, S., et Lebas, J. M., C. R. Hebd. Séan. Acad. Sci. Paris 272B (1971) 224-227. Spectrochim. Acta 27A (1971) 1337.

[6] Hadni, A., Wyncke, B., Morlot, G. et Gerbaux, X., J. Chem. Phys. 51 (1969) 3514.

[7] Wyncke, B., Hadni, A. et Gerbaux, X., J. Physique 31 (1970) 893.

[8] Cox, E. G., Rev. Mod. Phys. (1958) 159. 